\title{
Assessment of Knowledge and Perception Towards Modern Contraceptives Use Among Women of Reproductive Age in Mtwivila, Tanzania
}

\author{
Peter Elia Mosha ${ }^{*}$, Christina Abel Mgimwa, Stanslaus Mbonea Msuya
}

Institute of Rural Development Planning, Dodoma, Tanzania

Email address:

pmosha@irdp.ac.tz (P. E. Mosha)

${ }^{*}$ Corresponding author

\section{To cite this article:}

Peter Elia Mosha, Christina Abel Mgimwa, Stanslaus Mbonea Msuya. Assessment of Knowledge and Perception Towards Modern Contraceptives Use Among Women of Reproductive Age in Mtwivila, Tanzania. Science Journal of Public Health.

Vol. 5, No. 4, 2017, pp. 335-340. doi: 10.11648/j.sjph.20170504.19

Received: May 4, 2017; Accepted: May 17, 2017; Published: July 13, 2017

\begin{abstract}
This study was conducted in 2014 in Mtwivila, Tanzania. The objective of the study was to assess knowledge, perception and factors associated with use of modern contraceptive use among women. Simple random sampling technique was used to select a representative sample of 96 women aged 15-49 years. Data were collected through interviews, focus group discussions and documentary reviews. Findings show that knowledge of modern FP was almost universal (100\% percent), although 79 (82.3 percent) claimed to have been using modern contraceptives methods. Much smaller proportion (17.7 percent) reported not to be using modern contraceptives during the time of interview. Most of the respondents (96\%) had positive attitude on modern contraceptive use as 96 of all interviewed women stated contraceptives were effective for birth control and that they would recommend to a friend or a relative the use of modern contraceptive. However, $28 \%$ women reported having stopped using modern contraceptive of which $25 \%$ due to side effects. Chi-square confirmed the association of education, sources of information about contraceptives, occupation, household income, distance from the health center and husband's involvement with the use of contraceptives among women. It is concluded that there is a good level of knowledge and a positive perception towards contraceptive use among women of reproductive age. Men involvement during sensitization on family planning would be an effective strategy toward sexual and reproductive health promotion.
\end{abstract}

Keywords: Family Planning, Contraceptives Methods, Tanzania

\section{Introduction}

Whenever human reproduction is left unchecked, it results into high birth rates [1]. Indeed, uncontrolled births can destroy a nation's development aspirations and prevent its people from enjoying improved standard of living. Family Planning (FP) in which the major component is the use of contraceptive methods is a key fundamental of health services whose benefits are health and wellbeing of women, men, children, families, and their communities [1]. The concept of contraceptive use as used in this study took the definition from WHO. The WHO (2015) defined the contraceptive as use of a product or medical procedure that interferes with reproduction from acts of sexual intercourse. Contraceptive methods can be divided into two categories: traditional and modern. Modern contraceptives include oral contraceptives, intrauterine devices (IUDs), female and male sterilization, injections, condoms and the diaphragm. Other practices, which have a direct impact on fertility that have been used include prolonged breast feeding and postpartum sexual abstinence, which are probably used child survival and child spacing rather than for limiting family size.

In 2015, the United Nations report on Trends in Contraceptive Use Worldwide, it was reported that contraceptive use has increased in several parts around of the world, including Asia and Latin America [2]. Similarly, the report showed that the contraceptive use continues to be low in sub-Saharan Africa. Worldwide, the use of modern 
contraceptive has risen slightly, from $54 \%$ in 1990 to $57.4 \%$ in 2014. Looking at Africa it was revealed that contraception went from $23.6 \%$ to $27.6 \%$, in Asia from $60.9 \%$ to $61.6 \%$, and in Latin America and the Caribbean it rose from $66.7 \%$ to $67.0 \%$ [2].

Many sub-Saharan African (SSA) countries have persistently high rates of unmet need for family Planning [3] and low rates of contraceptive use. The low levels of contraceptive use in SSA are results of both the limited capacity of the health system and the structure within which contraceptive services are provided. There are a number of factors are known to affect utilization of contraceptives. These include risk perception, insufficient knowledge needed to make informed choices, opposition from male partners, and health service limitations [4].

In Tanzania, use of any method was reported as $26 \%$ of married women in 2004-05 but a slightly higher rate (34\%) was recorded in 2010 [5]. Low rate of use of contraceptives in Tanzania are due to shortage in some of the available and used methods, limited numbers of family methods' skilled service providers, insufficient/ low level skills of these staff, low education to users especially on modern contraceptive methods, fear of social disapproval, side effects posed by these methods to users' health, partner's disapproval, preference for large family sizes and other cultural concerns $[6,7,8]$. In Iringa, rates of population growth and levels of fertility are among the highest in the country and exacerbate many development problems. This paper aims at (i) examining knowledge and perception on the use of modern contraceptives methods among women of reproductive age, and (ii) identifying the factors influencing use of contraceptives.

\section{Method}

\subsection{Study Area}

The study was carried out in Mtwivila ward, Iringa Municipality in July 2014. The Municipality is located in the Southern highlands of Tanzania lying along $7^{\circ} 49 \mathrm{~S}$ and $35^{\circ} 39$ E.

\subsection{Study Design}

The study adopted a cross-sectional survey in collecting data from women of reproductive age (15 - 49 years). Data collection involved interviews, focus group discussions, and documentary reviews from official health documents and Demographic Health Survey 2010 book. A total of 96 randomly selected women were enrolled in the study. Since the total number of all women of reproductive age (15 to 49 years) in Mtwivila was not known, proportional sampling formula (1) presented below was used to determine the optimal sample size.

$$
n=\frac{\left(Z_{\alpha / 2}\right)^{2} \cdot P(1-P)}{\lambda^{2}}
$$

Where $n=$ sample size $(\mathrm{Z} \alpha / 2)^{2}=$ is the critical value $95 \%=(1.96) p=$ proportion of users. In this case, we adopted $p=50 \%=0.5$ and $\lambda^{2}=$ maximum error $=10 \%=0.1$

$$
n=\frac{(1.96)^{2} 0.5(1-0.5)}{0.1^{2}}=96
$$

The sampled respondents were 96 women of reproductive age enrolled in this study.

\subsection{Data Analysis}

Collected data were carefully coded and entered into IBM SPSS Statistics and then cleaned before they were analyzed. Data were analyzed for descriptive statistics including frequencies, mean, and cross tabulation. Chi-square test was performed to assess whether levels of income, occupation, education, distance to the nearest health center, partners approval, sources of information and household size have had an association with the use of modern contraceptives.

\section{Results and Discussion}

This section presents demographic and social characteristics of the women who were involved in the study. The section provides details of the knowledge of women on modern contraceptives, the perception of women of reproductive age in the study area and then narrated the factors influencing the use of modern contraceptives.

\subsection{Social-demographic Characteristics}

Table 1 shows a distribution of important demographic characteristics of the study participants. Women who were involved in the study were mainly aged $30-45$ years $(47.9 \%)$ and married (55.2\%). These two characteristics (age higher than 30 years and having at least secondary education), when combined together, had a positive influence on the use of contraceptives. Socio- demographic data are among the major factors known to influence individual's decisions on contraception and fertility $[9,10,11]$.

In this study, majority of the respondents $(79.3 \%)$ were in monogamous marriages. Studies show that individuals that are monogamous have a positive attitude over the use of contraceptives and have better chances to use contraceptives than those in a polygamous marriage $[12,13]$. Table 1 also indicates that participant's annual income ranged from USD 120 to 2,886 with an average income of USD 566.4 (with a S. E. mean of USD 43.15), household size ranging from 1 11 people with an average household size of 5 people. Again the study revealed that distance of participants to the nearest health facility ranges from $1 \mathrm{Km}$ to $4 \mathrm{Km}$ with an average distance of $1.92 \mathrm{Km}$. These variables are also considered to have an effect on the attitude and knowledge of a woman to use or not to use modern contraceptives as they influence woman health seeking behavior [14]. 
Table 1. Distributions of respondent by socio-demographic characteristics.

\begin{tabular}{lll}
\hline Demographic characteristics & n & \% \\
\hline Age(Years) & 19 & 19.8 \\
$15-30$ & 46 & 47.9 \\
$30-45$ & 31 & 32.3 \\
$46-49$ & & \\
Marital status & 38 & 39.6 \\
Single & 53 & 55.2 \\
Married & 5 & 5.2 \\
Widow & & \\
Education level & 30 & 31.2 \\
Primary level & 54 & 56.6 \\
Secondary level & 12 & 12.5 \\
College and above & & \\
Household Size & 58 & 60.4 \\
$0-5$ & 36 & 37.5 \\
6-10 & 2 & 2.1 \\
More than 10 & & \\
Occupation of respondent & 19 & 19.8 \\
Student & 28 & 29.2 \\
Peasant & 42 & 43.5 \\
Business & 7 & 7.3 \\
Teacher & & \\
Household Income (USD $\left.{ }^{* *}\right)$ & 14 & 14.6 \\
Low & 67 & 69.8 \\
Medium & 15 & 15.6 \\
High & & \\
\hline
\end{tabular}

** Where low $=(0-300.6)$ Medium $(300.6-601.3)$ High $=($ More 601.3 $)$ July 2014 exchange rate lusd for 1663.05 TZS

\subsection{Knowledge Among Women of Reproductive Age Toward Modern Contraceptives Methods}

Analysis of the data revealed knowledge of contraceptives was universal. Women interviewed during the study had heard about modern contraceptive methods, whereby the most commonly known modern method was pills (97.9\%) and male condoms $(97.9 \%)$. Further, inquiry on the major methods of contraceptives used showed pills and male condoms ranked higher $(97.9 \%)$ followed by injectable $(22.7 \%)$.

Preference of injection, intrauterine device (IUD), and Norplant method was also noted during an interview with the nurse and doctors as a key informants. Regarding source of information about the modern contraceptives, the findings show that the major sources of information were the hospital, media (radio, television and social network), schools, and friends. Further, women interviewed were asked to respond to types of contraceptives they knew and the results are presented in Table 2. These results show that most women are aware of at least one or more contraceptives, implying that they have a number of choices between contraceptives if they are willing to use. Discussing more on source of information about modern contraceptive use, it was noted that time taken and distance to the health facilities influenced the use of contraceptives positively.
Table 2. Knowledge of contraceptives among respondents $(n=96)$.

\begin{tabular}{lll}
\hline Contraceptive method & $\mathbf{n}$ & $\mathbf{\%}$ \\
\hline Ever heard modern contraceptives & 96 & 100.0 \\
Types of modern contraceptives methods Known* & & \\
Pills & 94 & 97.9 \\
Injection & 76 & 79.2 \\
Male condom & 94 & 97.9 \\
Norplant & 52 & 54.2 \\
IUD & 19 & 19.8 \\
Number of modern contraceptives methods known** & & \\
Low 1-2 & 7 & 7.3 \\
Medium 3-4 & 49 & 51.0 \\
High 4-5 & 40 & 41.7 \\
Sources of information on modern contraceptives* & & \\
Radio & 38 & 39.6 \\
Hospital & 57 & 59.4 \\
Schools & 20 & 20.8 \\
Friends & 21 & 21.9 \\
\hline * Data based on multiple responses ** where low = $(0-1)$ & medium & $=(2-3)$ \\
and high = (4-5) &
\end{tabular}

Table 3. Preference of contraceptives among respondents $(n=96)$.

\begin{tabular}{|c|c|c|}
\hline Variable & $\mathbf{n}$ & $\%$ \\
\hline \multicolumn{3}{|l|}{ Contraceptives used } \\
\hline Pills & 27 & 34.2 \\
\hline Injection & 12 & 15.2 \\
\hline Male condom & 24 & 30.4 \\
\hline Norplant & 16 & 20.3 \\
\hline \multicolumn{3}{|c|}{ If currently using Modern contraceptives } \\
\hline Yes & 50 & 63.3 \\
\hline No & 29 & 36.7 \\
\hline \multicolumn{3}{|c|}{ Reasons for Dropping from using Modern Contraceptives } \\
\hline Desire to have more children & 12 & 42.9 \\
\hline Experienced side effects & 7 & 25.0 \\
\hline Menopausal started & 5 & 17.9 \\
\hline Partner died (widow) & 4 & 14.3 \\
\hline \multicolumn{3}{|c|}{ If ever shifted from one type of contraceptive to another } \\
\hline Yes & 15 & 19.0 \\
\hline No & 64 & 81.0 \\
\hline \multicolumn{3}{|c|}{$\begin{array}{l}\text { Reasons for Shifting from one contraceptive to another } \\
\text { contraceptive }\end{array}$} \\
\hline Experienced side effects & 7 & 46.7 \\
\hline Use for Long period of time & 8 & 53.3 \\
\hline \multicolumn{3}{|c|}{ Type of Contraceptive newly adopted } \\
\hline Injection to Male condom & 1 & 6.7 \\
\hline Pills to Male Condom & 6 & 40.0 \\
\hline Pills to Injection & 3 & 20.0 \\
\hline Injection to Norplant & 1 & 6.7 \\
\hline Pills to Norplant & 3 & 20.0 \\
\hline Norplant to Male condoms & 1 & 6.7 \\
\hline
\end{tabular}

Table 3 shows results of the preference of contraceptive methods. Findings show that pills are more used followed by male condoms. There was no one mention on use of IUD.

Despite the fact that modern contraceptives method reduce fertility rate and give women time to rest after birth, out of all participants interviewed and ever heard of modern contraceptive, only $63.3 \%$ were currently using at least one method of contraceptives. The participants who dropped from using modern contraceptives were requested to share why they stopped, and almost half of all who responded to the question mentioned desire to have more children to be a major reason for dropping (42.9\%), while others mentioned their reasons to be experiencing side effects, menopausal and 
death of husband. Results presented in Table 3, showed very few women had ever shifted from using one contraceptive to another, and they shifted from pills to male condoms $(40.0 \%)$ due to experiencing the side effect or having used the methods for a long time that they desired a change. Studies have indicated experiencing sides effect is one of the major factors for those who are not using fail to adopt the use of modern contraceptive [10, 15].

Having knowledge about fertility control is an important stage towards attaining access to and then using an appropriate contraceptive method timely and in an effective manner [5]. It is also clear from the data that women interviewed accessed information about contraceptive methods from various sources, 57 (59.4\%) from hospitals in various visit, 38 (39.6\%) through radio programs, 20 (20.8\%) through schools for those who were still schooling at various levels, and $20(21.9 \%)$ heard of contraceptives from friends. The respondents were asked about the advantages and disadvantages of using modern contraceptives. It was noticed that although all women universally have heard of contraceptives, not all of them were using (see Table 3). Table 4 presents advantages and disadvantages for using modern contraceptives as reported by Mtwivila women.

Table 4. Advantages and disadvantages for using modern contraceptives ( $n$ $=96) *$.

\begin{tabular}{lll}
\hline Variable & n & \% \\
\hline Advantages for Using Modern & & \\
Contraceptives & 43 & \\
Prevent unwanted pregnancy & 27 & 38.3 \\
Space for children & 6 & 30.3 \\
Freedom to enjoy sex & 17 & 6.7 \\
Prevent diseases & 10 & 19.1 \\
Easy to use & 6 & 11.2 \\
Create confidence & 2 & 6.7 \\
Reduce birth rate & 5 & 2.2 \\
More effective & & 5.6 \\
Disadvantages for using & & \\
Contraceptives & 11 & \\
Lead to unwanted pregnancy & 7 & 17.2 \\
Lead to diseases & 30 & 10.9 \\
Lead to irregular bleeding & 8 & 46.9 \\
Lead to stress and fear & 12 & 12.5 \\
Lead to sterility & 13 & 18.8 \\
Weight gain & 20.3 \\
\hline
\end{tabular}

Data based on multiple responses

Mentioning of advantages of modern contraceptives among married women of reproductive age and being able to highlight some negative opinions that worry users is one of the indicators as of how much these women of Mtwivila are knowledgeable about the modern contraceptives.

\subsection{Perception towards Modern Contraceptives Use Among Women of Reproductive Age}

Perception factors discussed here are partner's approval, benefits of using contraceptives, opinion of recommending modern contraceptives to a friend, intention to use contraceptives in future and partner opinion on the importance of modern contraceptives. During focus discussion, one respondent said;

"I started using contraceptive after giving birth to my second child. I used pills without my husband approval for a while until when he found these pills in my handbag. At first, he was furious, but after explaining the importance and him consulting some of his friends we agreed that I should keep on using, though he had fear of side effects, the possibility of me cheating with other men and even possibility of not conceiving in the future."

The narratives support results of quantitative analysis of perceptions to contraceptive use. The narratives also indicate that women and even their partners have negative perceptions even though some had time to discuss contraceptive issues.

Table 5. Perception of respondents towards modern contraceptives $(n=96)$.

\begin{tabular}{lll}
\hline Variable & $\mathbf{n}$ & $\mathbf{\%}$ \\
\hline Benefits of modern contraceptives outweigh negative & & \\
effects $(\mathrm{n}=93)$ & 34 & 36.6 \\
Yes & 23 & 24.7 \\
No & 36 & 38.7 \\
Not Sure & & \\
If would recommend the use of modern contraceptive & & \\
to a friend $(\mathrm{n}=80)$ & 69 & 86.3 \\
Yes & 11 & 13.7 \\
No & & \\
If you would use modern contraceptives use in future & & 23.4 \\
( $\mathrm{n}=17$ ) & 4 & 76.5 \\
Yes & 13 & \\
No & & \\
If you would discuss with husband on modern & & 58.3 \\
contraceptives (n=96) & 56 & 41.7 \\
Yes & 40 & \\
No & & \\
Husband thinks that modern contraceptives are & 53 & 55.2 \\
important (n = 96) & 43 & 44.8 \\
Yes & & \\
No & & \\
\hline
\end{tabular}

Despite the side effects and even some women dropping from the use of modern contraceptives, Table 5 shows that some of the respondents had a positive perception towards the use of modern contraceptives. Over one third (36.6\%) of those who were using modern contraceptives during the time of interview argued that the benefits of the contraceptives outweigh the side effects and thus they would recommend to a friend to use contraceptives (86.3\%). Furthermore, 58.3\% of the participants confirmed to sharing with their husband discussion about the use of modern contraceptive where $55.2 \%$ of the respondents stated that their husbands approved use of contraceptives. Nevertheless, over forty percent of women $(41.7 \%)$ indicated that it was not possible to discuss contraceptive issues with their partners. Indeed, many studies have confirmed that most of African husband's attitude affect women decision towards modern contraceptives. The observed high proportion of women not being able to discuss the use of contraceptives with their husbands calls for the need to reach out to more women and men in Mtwivila to help them realize the importance of family planning along with the recommended methods of family planning. 


\subsection{Factors Influencing use of Modern Contraceptive Among Women of Reproductive Age}

Cultural, demographic and socio-economic factors such as education, sources of information, the occupation of the participant, household size, household annual income size, distance to the nearest health centre and discussion with husband were assessed if they had any influence on the use of the contraceptives among women. Results show that there is a significant association between the education level and the use of contraceptives. Use of modern contraceptives was associated with attaining secondary education or higher compared to those with primary education ( $\left.\chi^{2}=13.15, p=0.001\right)$. Further, results show a significant relationship between the use of contraceptives and source of information $\left(\chi^{2}=18.94, p=0.00\right)$. Health facility contributed women to use modern contraceptives, whereby $41.9 \%$ of the respondents obtained information about modern contraceptives from the hospitals. Further, child spacing, avoiding unwanted pregnancies and having time for other economic activities were the main drivers behind use of modern contraceptives. It was further revealed that household annual income, discussion of contraceptive issues with husband and husband's approval on the use of contraceptives were associated with increased chances of a woman use of modern contraceptive as presented in Table 6 below.

Table 6. Factors influencing the use of modern contraceptives among women of reproductive age.

\begin{tabular}{|c|c|c|c|c|}
\hline Variables & $\begin{array}{l}\text { use of } \\
\text { contraceptives }\end{array}$ & percent $\%$ & $\chi^{2}$ & P-value \\
\hline \multicolumn{5}{|c|}{ Level of education } \\
\hline Primary level & 30 & 31.2 & & \\
\hline Secondary level & 54 & 56.2 & 13.15 & $0.01 *$ \\
\hline College level & 12 & 12.5 & & \\
\hline \multicolumn{5}{|c|}{ Source of information } \\
\hline Radio & 38 & 27.9 & & \\
\hline Hospital & 57 & 41.9 & 18.94 & $0.02 *$ \\
\hline School & 20 & 14.7 & & \\
\hline Friends & 21 & 15.4 & & \\
\hline \multicolumn{5}{|l|}{ Occupation } \\
\hline Student & 19 & 19.8 & & \\
\hline Peasant & 18 & 18.8 & 17.307 & $0.00 *$ \\
\hline Business & 42 & 43.8 & & \\
\hline Teacher & 17 & 17.7 & & \\
\hline \multicolumn{5}{|l|}{ Household size } \\
\hline $0-5$ & 48 & 60.8 & & \\
\hline $6-10$ & 29 & 36.7 & 7.240 & $0.027 *$ \\
\hline More than 10 & 2 & 2.5 & & \\
\hline \multicolumn{5}{|c|}{ Household Annual } \\
\hline \multicolumn{5}{|c|}{ Income (USD**) } \\
\hline Low & 7 & 8.9 & & \\
\hline Medium & 57 & 72.2 & 10.262 & $0.016^{*}$ \\
\hline High & 15 & 19.0 & & \\
\hline \multicolumn{5}{|c|}{ Distance to Nearest } \\
\hline \multicolumn{5}{|c|}{ Health centre $(\mathrm{km})$} \\
\hline $0-1$ & 15 & 19.0 & & \\
\hline $1.1-2$ & 56 & 70.9 & 1.71 & NS \\
\hline $2.1-3$ & 8 & 10.1 & & \\
\hline \multicolumn{5}{|c|}{ Partners' Approval } \\
\hline Yes & 53 & 67.1 & & \\
\hline No & 26 & 32.9 & 4.899 & $0.027 *$ \\
\hline
\end{tabular}

Where low income $=(0-300.65)$, Medium income $=(300.66-601.30)$ and High income $=($ more than 601.30) NS $=$ Non-significant $(p>0.05) *=$ significant $(\mathrm{p}<0.05) * *$ July 2014 exchange rate 1USD for 1663.05 TZS

\section{Conclusions and Recommendations}

Knowledge of modern contraceptives was found to be high as majority of respondents knew at least three methods of contraceptives. Health facilities have significant influence on the use of modern contraceptives. Generally, majority of the respondents had positive attitudes on use of contraceptives. Male condom, pills, and injection were the most known contraceptive methods. Further, the study has clearly shown that education level and occupation of the respondents have significant influence on the use of contraceptives. Knowledge and a positive perception suggest increased use of contraceptives. Since men involvement in family planning and use of contraceptives was low, it is recommended that health agents should ensure inclusion of men while communicating messages on reproductive health. Further, health education in schools and college should be given due consideration and communicated effectively as it has a significantly positive effect on the use of contraceptives.

\section{References}

[1] Michael, E. J. (2012). Use of contraceptives methods among women in stable marital relations attending health facilities in Kahama district, Shinyanga region, Tanzania.

[2] United Nations (2015). Trends in Contraceptive Use Worldwide, (ST/ESA/SER. A/349).

[3] Westoff, C. F. (2001). Unmet Need at the End of the Century. DHS Comparative Reports No. 1. Calverton, Maryland: ORC Macro.

[4] Aryeetey, R., Kotoh, A. M \& Hindin, M. J. (2010). Knowledge, Perceptions and Ever Use of Modern Contraception among Women in the Ga East District, Ghana. African Journal of Reproductive Health December 2010; 14 (4): 27.

[5] United Republic of Tanzania (2010). Tanzania Demographic and Health Survey (TDHS), National Bureau of Statistics, Dar es Salaam, Tanzania, 1-451.

[6] Kabagenyi, A., Jennings, L., Reid, A., Nalwadda, G., Ntozi, J., \& Atuyambe, L. (2014). Barriers to male involvement in contraceptive uptake and reproductive health services: a qualitative study of men and women's perceptions in two rural districts in Uganda. Reproductive Health DOI: 10.1186/17424755-11-21.

[7] URT (2010). Tanzania Bureau of Statistics; Demographic Health Survey, Final Report 2010.

[8] Cleland, J., Bernstein, S., Ezeh, A. I., Faundes, A., Glasier, A \& Innis, J. (2006). Family Planning: The Unfinished Agenda, Journal Paper, Sexual and Reproductive Health, 3: 1-18.

[9] Dabral, S \& Malik, S. L. (2004). A demographic study of Gujjars of Delhi: IV. Kap of family planning. J. Hum. Ecol., 16 (4): 231-237.

[10] Igwegbe, A., Ugboaja, J., \& Monago, E. (2009). Prevalence and determinants of unmet need for family planning in Nnewi, South-east Nigeria. Int. J. Medic. Med. Sci., 1 (8): 325-329. 
[11] Burke, H. M. \& Ambasa-Shisanya, C. (2011). A qualitative study of reasons for discontinuation of injectable contraceptives among users and salient reference groups in Kenya. Afr. J. Reprod, Health, 15 (2): 67-78.

[12] Duze, M. \& Mohamed, Z. I. (2006). Male knowledge, attitudes and family planning practices in northern Nigeria. Afr. J. Reprod. Health, 10 (3): 53-65.

[13] Lwelamira, J., Mnyamagola, G \& Msaki. M. M. (2012). Knowledge, Attitude and Practice (KAP) Towards Modern Contraceptives among Married Women of Reproductive Age in Mpwapwa District, Central Tanzania. Maxwell Scientific
Organization. Current Research Journal of Social Sciences 4 (3): $235-24$

[14] Amin, R., Shah, N. M \& Becker, S. (2010). Socioeconomic factors differentiating maternal and child health-seeking behaviour in rural Bangladesh: A cross-sectional analysis. Int. J. Equity Health, 9: 9.

[15] Marchant, T., Mushi, A. K., Nathan, R., Mukasa, O., Abdulla, S., Lengeler, C \& Schellenerg, A. (2004). Planning a family: priorities and concerns in Rural Tanzania. African Journal of Reproductive Health. 8 (2): 111-124. 